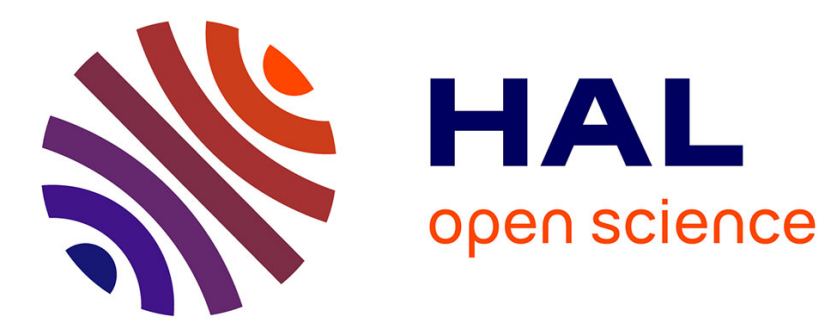

\title{
Networked control: taking into account sample period variations and actuators saturation
}

\author{
Alexandre Seuret, Joâo Manoel Gomes da Silva Gomes da Silva
}

\section{To cite this version:}

Alexandre Seuret, Joâo Manoel Gomes da Silva Gomes da Silva. Networked control: taking into account sample period variations and actuators saturation. IFAC WC 2011 - 18th IFAC World Congress, Aug 2011, Milan, Italy. pp.6. hal-00584384

\section{HAL Id: hal-00584384 https://hal.science/hal-00584384}

Submitted on 8 Apr 2011

HAL is a multi-disciplinary open access archive for the deposit and dissemination of scientific research documents, whether they are published or not. The documents may come from teaching and research institutions in France or abroad, or from public or private research centers.
L'archive ouverte pluridisciplinaire HAL, est destinée au dépôt et à la diffusion de documents scientifiques de niveau recherche, publiés ou non, émanant des établissements d'enseignement et de recherche français ou étrangers, des laboratoires publics ou privés. 


\title{
Networked control: taking into account sample period variations and actuators saturation
}

\author{
Alexandre Seuret* Joao M. Gomes Da Silva Jr. ${ }^{* *}$ \\ ${ }^{*}$ NeCS Team, Automatic Control Department of Grenoble GIPSA-Lab, UMR \\ CNRS 5216. E-mail: alexandre.seuret@gipsa-lab.inpg.fr \\ ** UFRGS - Department of Electrical Engineering, Porto Alegre-RS, Brazil. \\ E-mail: jmgomes@ece.ufrgs.br
}

\begin{abstract}
This paper exposes a novel method to cope with the stabilization of networked control systems with asynchronous sampling and actuators saturations. The constructive stabilization criterion is expressed in terms of linear matrices inequalities using the continuous-time model of the systems. However the stability analysis of the closed-loop system is based on the discrete-time Lyapunov Theorem. An example shows that the conservatism of the conditions has been reduced with respect to the literature.
\end{abstract}

\section{INTRODUCTION}

In the last decades, a large attention has been taken to Networked Control Systems (NCS) (see Hespanha et al. (2007); Zampieri (2008)). Such systems are controlled systems containing several distributed plants which are connected through a communication network. In such applications, a heavy temporary load of computation in a processor can corrupt the sampling period of a certain controller. On the other side, the sampling period can be scheduled in the design in order to avoid this load. In both cases, the variations of the sampling period will affect the stability properties of such systems. Another phenomenon, which has been widely investigated concerns stability under packet losses. In wireless networks, a transmission of data packets is not always guaranteed. Some packets can be lost during the transmission. The objective is thus to guarantee the stability even if some packet are lost in the communication. It is thus an important issue to develop robust stability conditions with respect to the variations of sampling period.

The variation of the sampling period in a networked control loop can be modeled by a time-varying delay on the plant control input while considering a continuous-time dynamics. In this case, the delay variation rate between two sampling instants is equal to one. Thus, a fundamental problem regards the determination of the bounds on the delay variation (corresponding to the sampling period jitter), for which the stability of the closedloop system can be kept. On the other hand, for physical or security reasons, actuators cannot apply unlimited signals to the controlled plant. This fact, makes control saturation another possible source of instability and performance degradation. Hence the problem of stabilizing a networked control system taking into account the variations on the sampling period as well as the control signal constraints, can be seen as a problem of stabilizing a continuous-time system presenting a saturating delayed input.

In the last decade, many techniques have been proposed in the literature to deal with the stabilization of time-delay systems

\footnotetext{
‡ This work was supported by the European project FeedNetBack (http://www.feednetback.eu/). The work of J.M. Gomes da Silva Jr. is supported by CNPq (Brazil) grants PQ: 306210/2009-6 and Ed. UNIV: 480756/2009-0
}

subject to actuator saturation. In this context, we can cite, for instance: Oucheriah (1996) and Niculescu et al. (1996), where globally stabilizing control laws are proposed, and Tarbouriech and Gomes da Silva Jr. (2000), Cao et al. (2002) and Fridman et al. (2004), where the regional stabilization problem is considered. These works are mainly concerned by state delayed systems. In this context, the stabilization conditions consider delay independent approaches (which allow to address timevarying delays, but in a possible conservative way) and delay dependent conditions considering fixed delays. Recently, in Zhang et al. (2008) and Gomes da Silva Jr. et al. (2010), results considering systems presenting time-variyng delays on the states have been proposed. On the other hand, we can note a lack of results considering input delays, and, in particular, time-varying ones. In Tarbouriech et al. (2003) and Gomes da Silva Jr. and Tarbouriech (2005), anti-windup techniques are proposed for systems with fixed input delays. In Fridman et al. (2004), the particular case of sample data problem is considered from an input time-delay perspective. This result is based on the the polytopic formulation from Cao et al. (2002) which increases the number of LMIs to solve. Moreover this result from Fridman et al. (2004) on stability and stabilization of sampleddata systems has been improved, for instance in Naghshtabrizi et al. (2008) and Seuret (2011). Then there is a need to develop a more accurate stabilization criteria for sampled and saturated controlled systems.

In this paper we are interested in the problem of stabilizing a networked control system taking into account the possible variations on the sample period (due to packet losses in the network) and also the fact that the signals provided by the actuators are bounded (i.e saturating plant inputs). With this aim, the following section describes the problem formulation. Section 3 presents some preliminary lemmas on LMI manipulations, on the generalized sector condition and a preliminary theorem on the stability of asynchronous sampling. Then Sections 4 and 5 respectively expose a theorem on the local stabilization of systems with sampled and saturated input and an optimization method. An example is showed in section 6 .

Notations. Throughout the article, the sets $\mathbb{N}, \mathbb{R}^{+}, \mathbb{R}^{n}, \mathbb{R}^{n \times n}$, $\mathbb{S}^{n}$ and $\mathbb{K}_{T}$ denote respectively the set of positive integers, pos- 
itive scalars, $n$-dimensional vectors, $n \times n$ matrices, symmetric matrices of $\mathbb{R}^{n \times n}$ and the set of differentiable function from an interval $\mathscr{I} \subset[0, T]$ to $\mathbb{R}^{n}$. For a given positive scalar, $T_{2}$, define the $\mathbb{K}$ as the set of continuous functions from $\left[0, T_{2}\right]$ to $\mathbb{R}^{n}$. The notation $\|\cdot\|$ stands for the Euclidean norm. The superscript ' $T$ ' stands for matrix transposition. The notation $P>0$ for $P \in \mathbb{S}^{n}$ means that $P$ is positive definite. For any matrix $A \in \mathbb{R}^{n \times n}$ and vector $x \in \mathbb{R}^{n}$, the notations $A_{j}$ and $x_{j}$ denote, respectively, the $j^{\text {th }}$ line of matrix $A$ and the $j^{\text {th }}$ component of vector $x$. The term $\mathrm{He}\{A\}>0$ refers to $A+A^{T}>0$. The symbols $I$ and 0 represent the identity and the zero matrices of appropriate dimension.

\section{PROBLEM FORMULATION}

Let $\left\{t_{k}\right\}_{k \in \mathbb{N}}$ be an increasing sequence of positive scalars such that $\bigcup_{k \in \mathbb{N}}\left[t_{k}, t_{k+1}[=[0,+\infty[\right.$. Assume that there exist two positive scalars $\bar{T}_{1} \leq \bar{T}_{2}$ such that the difference between two successive sampling instants $T_{k}=t_{k+1}-t_{k}$ satisfies

$$
\forall k \in \mathbb{N}, \quad 0 \leq \bar{T}_{1} \leq T_{k} \leq \bar{T}_{2} .
$$

Consider the linear system with a sampled-data input

$$
\dot{x}(t)=A x(t)+B u\left(t_{k}\right),
$$

where $x \in \mathbb{R}^{n}$ and $u \in \mathbb{R}^{m}$ represent the state variable and the input vector. The matrices $A$ and $B$ are of appropriate dimension. We suppose that the input vector $u$ is subject to amplitude limitations defined as follows:

$$
\left|u_{i}\right| \leq u_{0 i}, \quad u_{0 i}>0, i=1, \ldots, m .
$$

Consider now a state feedback control law $u(t)=K x(t)$. Due to the control bounds defined in (3), the effective control signal to be applied to the system is given by

$$
u(t)=\operatorname{sat}\left(K x_{k}\right), t_{k} \leq t<t_{k+1},
$$

where $x_{k}=x\left(t_{k}\right)$ and the gain $K$ in $\mathbb{R}^{n \times m}$ is to be determined and $\left.u_{i}(t)=\operatorname{sat}\left(K_{i} x_{k}\right)=\operatorname{sign}\left(K_{i} x_{k}\right)\right) \min \left\{u_{0 i}, K_{i} x_{k}\right\}$ for $i=1, \ldots, m$. Hence, the closed-loop system reads

$$
\dot{x}(t)=A x(t)+B s a t\left(K x_{k}\right) .
$$

Note that system (5) can be in fact be represented by the following input delayed system:

$$
\dot{x}(t)=A x(t)+B s a t(K x(t-\tau(t)))
$$

with $\tau(t)=t-t_{k}$ and $\tau(t) \leq \bar{T}_{2}$. Furthermore, between $t_{k}$ and $t_{k+1}$, one has $\dot{\tau}(t)=1$. Integrating the differential equation (2) with the control law (4) over a sampling period, the dynamics of the system satisfies, for all $t \in\left[t_{k}, t_{k+1}\right]$

$$
\begin{aligned}
& \dot{x}\left(t_{k}+\tau(t)\right)=\tilde{A}(\tau(t)) x_{k}+\tilde{B}(\tau(t)) \operatorname{sat}\left(K x_{k}\right), \\
& \tilde{A}(\tau(t))=e^{A \tau(t)}, \tilde{B}(\tau(t))=\int_{0}^{\tau(t)} e^{A(\tau(t)-\theta)} d \theta B .
\end{aligned}
$$

This equality leads naturally to the introduction of a novel notation. Define, for all integer $k$, the function $\chi_{k}(\tau):\left[0, T_{k}\right] \rightarrow$ $\mathbb{R}^{n}$ such that for all $\tau$ in $\left[0, T_{k}\right], \chi_{k}(\tau)=x\left(t_{k}+\tau\right)$. It represents the trajectory of the sampled-data systems (2) in the interval

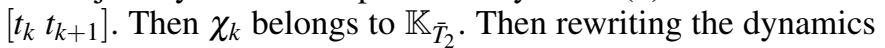
of the system using $\chi_{k} \in \mathbb{K}_{\bar{T}_{2}}$ and $\tau \in\left[0, T_{k}\right]$ leads to

$$
\begin{aligned}
& \chi_{k}(\tau)=x\left(t_{k}+\tau\right)=\tilde{A}(\tau) \chi_{k}(0)+\tilde{B}(\tau) \operatorname{sat}\left(K \chi_{k}(0)\right) \\
& \dot{\chi}_{k}(\tau)=\frac{d}{d \tau} \chi_{k}(\tau)=A \chi_{k}(\tau)+\operatorname{Bsat}\left(K \chi_{k}(0)\right)
\end{aligned}
$$

Although the system dynamics are considered to be linear, due to the control saturation, the closed-loop system is nonlinear. Hence, the determination of a global stabilizing controller is possible only when some stability assumptions are verified by the open-loop system $(u(t)=0)$ Lin and Saberi (1993). When this hypothesis is not verified, it is only possible to achieve semiglobal or local/regional stabilization. In this case, given a stabilizing matrix $K$, we associate a basin of attraction to the equilibrium point $x_{e}(t) \equiv 0$ of system (5). The basin of attraction corresponds to all initial conditions $x_{0} \in \mathbb{R}$ such that the corresponding trajectories of system (5) converge asymptotically to the origin Khalil (1996). Since the determination of the exact basin of attraction is practically impossible, a problem of interest is to ensure the asymptotic stability for a set of admissible initial conditions $x_{0}$.

Hence, from the considerations above, in this paper we are interested in studying the stabilization problems stated as follows.

(P1) Given $\bar{T}_{1}, \bar{T}_{2}$, find $K$ and a set of admissible initial conditions, as large as possible, for which the asymptotic stability of the closed-loop system is ensured.

(P1) Maximize the bound on the delay $\bar{T}_{2}$, for which the asymptotic stability of the closed-loop system can be ensured for some set of admissible initial conditions.

In the sequel, theoretical conditions that allow to address the stabilization problems above are proposed.

\section{ASYMPTOTIC STABILITY OF SATURATED AND SAMPLED-DATA SYSTEMS}

\subsection{Preliminaries}

Lemma 1. Let $P$ and $P_{0}$ be two positive definite matrices and $M$ being a nonsingular matrix. Define $\tilde{P}=M P M^{T}$. If

$$
\left[\begin{array}{cc}
-P_{0} & I \\
I & -M-M^{T}+\tilde{P}
\end{array}\right]<0
$$

then $P<P_{0}$.

Proof: If $P>0$, it follows from

$$
\left(P^{-1}-M\right) P\left(P^{-1}-M\right)^{T} \geq 0
$$

that $P^{-1} \geq\left(M^{T}+M-\tilde{P}\right)$ or $P \leq\left(M+M^{T}-\tilde{P}\right)^{-1}$. Hence, from Schur's complement, if (8) is verified, it follows that:

$$
P_{0}>\left(M+M^{T}-\tilde{P}\right)^{-1} \geq P,
$$

which implies that $P<P_{0}$.

\subsection{Generalized sector condition}

Define the following function

$$
\psi\left(K \chi_{k}(0)\right)=K \chi_{k}(0)-\operatorname{sat}\left(K \chi_{k}(0)\right)
$$

Note that, $\psi\left(K \chi_{k}(0)\right)$ corresponds to a decentralized deadzone nonlinearity. Considering the function $\psi\left(K \chi_{k}(0)\right)$, the closedloop system can be re-written as

$$
\dot{\chi}_{k}(\tau)=A \chi_{k}(\tau)+B K \chi_{k}(0)-B \psi\left(K \chi_{k}(0)\right) .
$$

Considering a matrix $G \in \mathbb{R}^{m \times n}$ and defining the following polyhedral set

$$
\mathscr{S} \triangleq\left\{x \in \mathbb{R}^{n} ;\left|\left(K_{i}-G_{i}\right) x\right| \leq u_{0 i}, i=1, \ldots, m\right\} .
$$

The following Lemma, from Gomes da Silva Jr. and Tarbouriech (2005), concerning the nonlinearity $\psi(K x)$ can be stated.

Lemma 2. Consider the function $\psi(K x)$ defined in (10) for any $x \in \mathbb{R}^{n}$. If $x \in \mathscr{S}$ then the relation

$$
\psi^{T}(K x) U[\psi(K x)-G x] \leq 0,
$$

is verified for any matrix $U \in \mathbb{R}^{m \times m}$ diagonal and positive definite. 
The result in Lemma 2 can be seen as a generalized sector condition. As will be seen in the sequel, differently from the classical sector condition (used for instance in Tarbouriech et al. (2003)), this condition will allow to obtain stability conditions directly in an LMI form.

Another instrumental result, needed in the sequel to devise the stabilization conditions, is given by the following section.

\subsection{Stability of sampled-data systems}

This section is motivated by the difference between the discrete and continuous-time Lyapunov theorems. As the problem of sampled-data systems is at the boundary of the discrete and the continuous-time theories, it is important to put in clear the difference between them. More especially, the main idea of this section consists in developing novel stability conditions for sampled-data systems, modelled in continuous-time, using the discrete-time Lyapunov theorem.

Theorem 1. Let $V: \mathbb{R}^{n} \rightarrow \mathbb{R}^{+}$be a function for which there exist real numbers $0<\mu_{1}<\mu_{2}$ and $p>0$ such that

$$
\forall(k, x) \in \mathbb{N} \times \mathbb{R}^{n}, \quad \mu_{1}\|x\|^{p} \leq V(x) \leq \mu_{2}\|x\|^{p} .
$$

The two following statements are equivalent.

(i) $\forall k \geq 0, \quad \Delta V(k)=V\left(\chi_{k}\left(T_{k}\right)\right)-V\left(\chi_{k}(0)\right)<0$;

(ii) For all $T_{k} \in\left[\begin{array}{ll}\bar{T}_{1} & \bar{T}_{2}\end{array}\right]$, there exists a continuous functional $V_{1}:\left[\begin{array}{ll}0 & T_{k}\end{array}\right] \times \mathbb{K}_{\bar{T}_{2}} \rightarrow \mathbb{R}$, differentiable over all intervals of the form $\left[0 T_{k}\right.$ [ which satisfies for all $k \geq 0$

$$
V_{1}\left(T_{k}, \chi_{k}(\cdot)\right)=V_{1}\left(0, \chi_{k}(\cdot)\right)
$$

and such that, for all $k>0$ and for all $\tau$ in $\left[0 T_{k}\right]$, the following inequality holds

$$
\mathscr{W}\left(\tau, \chi_{k}\right)=\frac{d}{d \tau}\left[V\left(\chi_{k}(\tau)\right)+V_{1}\left(\tau, \chi_{k}\right)\right]<0,
$$

Moreover, if one of these two statements is satisfied, the solutions of the system (2) with the sampled and saturated control law (4) are asymptotically stable.

Proof. Consider a positive integer $k, T_{k} \in\left[\bar{T}_{1} \bar{T}_{2}\right]$ and $\tau \in\left[0, T_{k}\right]$. Assume (ii) is satisfied. Integrating $\mathscr{W}$ over the interval $\left[0 T_{k}[\right.$ and assuming that (15) holds, this directly implies $\Delta V(k)<0$ and that (i) holds.

Assume now that (i) is satisfied. Inspired by Lemma 2 in Peet et al. (2009), consider the functional $V_{1}\left(\tau, \chi_{k}\right)=-V(x)+$ $\tau / T_{k} \Delta V(k)$. Indeed, $V_{1}$ is a functional since it is expressed with respect to $\Delta V(k)$ which depends on the function $\chi_{k}(0)$, $\chi_{k}\left(T_{k}\right)$ and $\chi_{k}(\tau)$ for all $\tau \in\left[\begin{array}{ll}0 & T_{k}\end{array}\right]$. By simple computations, it is easy to obtain that this functional satisfies (15) and that $\mathscr{W}\left(\tau, \chi_{k}\right)=\Delta V(k) / T_{k}$. Then $\mathscr{W}$ has the same sign as $\Delta V(k)$. This proves the equivalence between (i) and (ii).

From the discrete-time Lyapunov theorem, the equilibrium of the discrete-time system is asymptotically stable by noting that the inequality (i) above implies $V\left(\chi_{k}\left(T_{k}\right)\right)<V\left(\chi_{k}(0)\right)$ and consequently that $x\left(t_{k}\right)$ tends to zero as $k$ tends to infinity.

The end of the proof consists in ensuring that the solutions of the continuous-time system are not diverging within a sampling period. From (14), the inequality

$$
\begin{aligned}
& \left.\left.V\left(\chi_{k}(\tau)\right) \leq \mu_{2}\right)\left\|\tilde{A}(\tau) \chi_{k}(0)+\tilde{B}(\tau) \operatorname{sat}\left(K \chi_{k}(0)\right)\right\|\right)^{p} \\
& V(x) \leq \mu_{2}\left((\|\tilde{A}(\tau)\|)^{p}+(\|\tilde{B}(\tau) K\|)^{p}\right)\left(\left|\chi_{k}(0)\right|\right)^{p}
\end{aligned}
$$

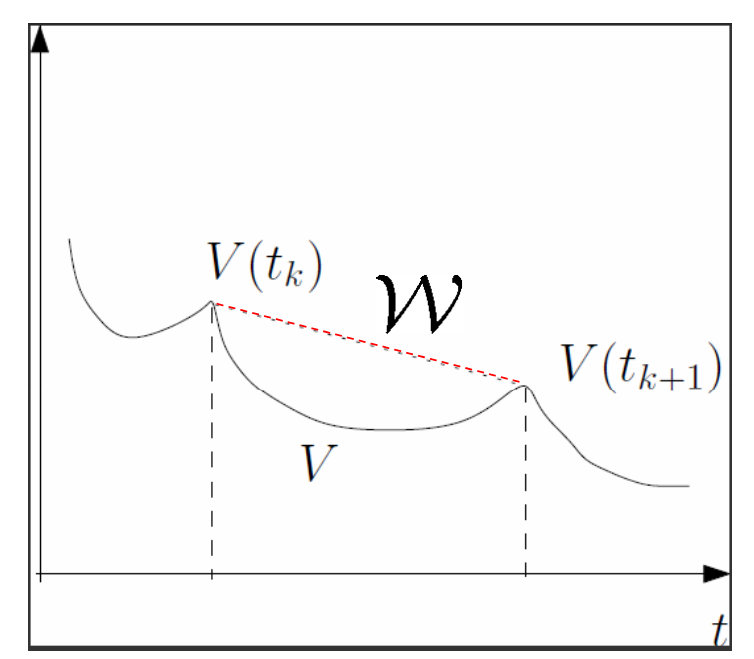

Fig. 1. Illustration of the proof of Theorem 1

is ensured, for all integer $k$, and $\tau \in\left[0, T_{k}\right]$. Noting that the functions $\tilde{A}($.$) and \tilde{B}($.$) from \left[0, \bar{T}_{2}\right]$ to $\mathbb{R}^{n \times n}$ are continuous and consequently bounded over $\left.\left[0, \bar{T}_{2}\right]\right]$, there exists a positive scalar $\mu_{m}$ such that,

$$
\forall \tau \in\left[0 \bar{T}_{2}\right], \quad(\|\tilde{A}(\tau)\|)^{p}+(\|\tilde{B}(\tau) K\|)^{p} \leq \mu_{m} .
$$

This ensures $V\left(\chi_{k}(\tau)\right) \leq \mu_{2} \mu_{m}\left\|\chi_{k}(0)\right\|^{p}$. This proves that the continuous Lyapunov function uniformly and exponentially tends to zero.

In the literature several articles have introduced functionals satisfying the requirements of Theorem 1 (see for instance Naghshtabrizi et al. (2008); Fridman (2010)). However, those results use of the Lyapunov-Krasovskii theorem which requires necessarily their positive definiteness. Moreover no direct relation between the discrete-time Lyapunov theorem $(\Delta V(k)<0)$ and the Lyapunov-Kravoskii theorem $\left(\dot{V}+\dot{V}_{1}<0\right)$ is provided. Theorem 1 proves that they are equivalent and, moreover, it relaxes the constraint on the positivity of the functional. The only requirement to ensure stability is on the discrete-time Lyapunov function $V$, and (15).

A graphical illustration of Theorem 1 is shown in Figure 1. The main idea remains in showing the equivalence between the conditions on the decreasing increment $\Delta V(k)=V\left(x_{t_{k+1}}\right)-$ $V\left(x_{t_{k}}\right)<0$ and the existence of a continuous functional $\mathscr{W}$ which coincides with the Lyapunov function $V$ at the sampling instants and which is strictly decreasing within all sampling intervals. The main contribution of Theorem 1 is that the introduction of the functional $\mathscr{W}$ allows the Lyapunov function $V$ to be locally increasing.

In the following, a theorem provides sufficient stability conditions for the cases of synchronous and asynchronous samplings.

\section{STABILIZATION OF SAMPLED DATA SYSTEMS UNDER INPUT SATURATION}

In this section, a constructive result regarding the asymptotic stability of the solutions of systems under saturation and sampled input is provided. The objective is to compute a control law and an associated set of initial conditions that makes the solutions of the closed loop system asymptotically stable. Based on the result of Theorem 1 , $t$ he following theorem is derived. 
Theorem 2. For given positive scalars $\bar{T}_{1}<\bar{T}_{2}$, assume that there exist, $\tilde{P}>0, \tilde{R}>0, \tilde{S}_{1} \tilde{X} \in \mathbb{S}^{n}$, a diagonal matrix $\tilde{U}>0$ in $\mathbb{S}^{m}, \tilde{K}, \tilde{G} \in \mathbb{R}^{m \times n}, \tilde{Y}, \tilde{S}_{2} \in \mathbb{R}^{n \times n}$ and $\tilde{N} \in \mathbb{R}^{(3 n+m) \times n}$ that satisfy, for $i=1,2$

$$
\begin{gathered}
\Psi^{1}\left(\bar{T}_{i}\right)=\tilde{\Pi}_{1}+\bar{T}_{i} \tilde{\Pi}_{2}+\bar{T}_{i} \tilde{\Pi}_{3}<0, \\
\Psi^{2}\left(\bar{T}_{i}\right)=\left[\begin{array}{cc}
\tilde{\Pi}_{1}-\bar{T}_{i} \tilde{\Pi}_{3} & \bar{T}_{i} \tilde{N} \\
* & -\bar{T}_{i} \tilde{R}
\end{array}\right]<0, \\
\Psi_{j}^{3}\left(u_{0 j}\right)=\left[\begin{array}{cc}
\tilde{P}(\tilde{K}-\tilde{G})_{j} \\
* & u_{0 j}^{2}
\end{array}\right] \geq 0, \quad \forall j=1, \ldots, m,
\end{gathered}
$$

with

$$
\begin{aligned}
\tilde{\Pi}_{1} & =\operatorname{He}\left\{M_{1}^{T} \tilde{P} M_{3}\right\}-M_{12}^{T} \tilde{S}_{1} M_{12}-\operatorname{He}\left\{\left(M_{2}^{T} \tilde{S}_{2}^{T}+\tilde{N}\right) M_{12}\right\} \\
& +\operatorname{He}\left\{\varepsilon M_{1}^{T}\left(A \tilde{Y} M_{1}+B \tilde{K} M_{2}-\tilde{Y} M_{3}-B \tilde{U} M_{4}\right)\right\} \\
& +\operatorname{He}\left\{M_{3}^{T}\left(A \tilde{Y} M_{1}+B \tilde{K} M_{2}-\tilde{Y} M_{3}-B \tilde{U} M_{4}\right)\right\} \\
& +\operatorname{He}\left\{M_{4}^{T} \tilde{G} M_{2}\right\}-2 M_{4}^{T} \tilde{U} M_{4} \\
\tilde{\Pi}_{2} & =M_{3}^{T} \tilde{R} M_{3}+\operatorname{He}\left\{M_{3}^{T}\left(\tilde{S}_{1} M_{12}+\tilde{S}_{2} M_{2}\right)\right\} \\
\tilde{\Pi}_{3} & =M_{2}^{T} \tilde{X} M_{2},
\end{aligned}
$$

where

$$
\begin{aligned}
& M_{0}=\left[\begin{array}{llll}
A & B K & -I-B
\end{array}\right], \\
& M_{1}=\left[\begin{array}{llll}
I & 0 & 0 & 0
\end{array}\right], \quad M_{2}=\left[\begin{array}{llll}
0 & I & 0 & 0
\end{array}\right], \\
& M_{3}=\left[\begin{array}{llll}
0 & 0 & I & 0
\end{array}\right], \quad M_{4}=\left[\begin{array}{llll}
0 & 0 & 0 & I
\end{array}\right],
\end{aligned}
$$

and where $M_{12}=M_{1}-M_{2}$.

then, for $K=W \tilde{Y}^{-1}$ and all initial conditions $x_{0}=x(0)$ belonging to the ellipsoidal set

$$
\mathscr{E}=\left\{x \in \mathbb{R}^{n}, \quad x^{T} P x \leq 1\right\},
$$

with $P=\tilde{Y}^{-T} \tilde{P} \tilde{Y}^{-1}$, the corresponding trajectories of system (5) converge asymptotically to the origin for any asynchronous sampling satisfying (1).

Remark 1. The previous matrices $M_{i}$ are not of the same dimension.

Proof. Introduce a quadratic Lyapunov function candidate defined by $V(x)=x^{T} P x$ for all $x \in \mathbb{R}^{n}$. The objective is then to prove that $\Delta V(k)=V\left(\chi_{k}\left(T_{k}\right)\right)-V\left(\chi_{k}(0)\right)<0$. Thanks to Theorem 1 , the objective is to design functionals $V_{1}$ which satisfies (15). A candidate of such type of functionals is defined for all $\tau \in\left[\begin{array}{ll}0 & T_{k}\end{array}\right]$

$$
\begin{aligned}
V_{1}\left(\tau, \chi_{k}\right)= & \left(T_{k}-\tau\right) \zeta_{k}^{T}(\tau)\left[S_{1} \zeta_{k}(\tau)+2 S_{2} \chi_{k}(0)\right] \\
& +\left(T_{k}-\tau\right) \int_{0}^{\tau} \dot{\chi}_{k}^{T}(\theta) R \dot{\chi}_{k}(\theta) d \theta \\
& +\left(T_{k}-\tau\right) \tau \chi_{k}^{T}(0) X \chi_{k}(0)
\end{aligned}
$$

where $\zeta_{k}(\tau)=\chi_{k}(\tau)-\chi_{k}(0)$. The first step of the proof focuses on the fact that the function $V$ and the functional $V_{1}$ satisfy Theorem (1). The function $V$ satisfies (14) since it is a quadratic Lyapunov function. The next part of the proof consists in ensuring (15). Since $\zeta_{k}(\tau)$ and $\left(T_{k}-\tau\right)$ tend to 0 as $\tau \rightarrow 0$ and $\tau \rightarrow T_{k}$, respectively, it follows that the limits of $V_{1}\left(\tau, \chi_{k}\right)$ tends to 0 as $\tau$ tends to 0 and $T_{k}$. The functional $V_{1}$ thus satisfies conditions (15). Moreover it is continuous at all sampling instants and differentiable for all $\tau \in\left[0 T_{k}\right.$ [. The rest of the proof consists in ensuring inequality (16). The following equality is obtained

$$
\begin{aligned}
\mathscr{W}\left(\tau, \chi_{k}\right) & =2 \chi_{k}^{T}(\tau) P \dot{\chi}_{k}(\tau)+\left(T_{k}-2 \tau\right) \chi_{k}^{T}(0) X \chi_{k}(0) \\
& -\zeta_{k}^{T}(\tau)\left[S_{1} \zeta_{k}(\tau)+2 S_{2} \chi_{k}(\tau)\right]-\int_{0}^{\tau} \dot{\chi}_{k}^{T}(\theta) R \dot{\chi}_{k}(\theta) d \theta \\
& +\left(T_{k}-\tau\right) \dot{\chi}_{k}^{T}(\tau)\left[R \dot{\chi}_{k}(\tau)+2 S_{1} \zeta_{k}(\tau)+2 S_{2} \chi_{k}(0)\right]
\end{aligned}
$$

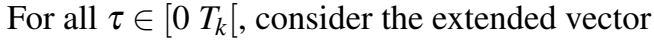

$$
\xi_{k}(\tau)=\left[\chi_{k}^{T}(\tau) \chi_{k}^{T}(0) \dot{\chi}_{k}^{T}(\tau) \psi^{T}\left(K \chi_{k}(0)\right)\right]^{T} .
$$

Introduce a matrix $N \in \mathbb{R}^{(3 n+m) \times n}$. Since $R$ is assumed to be positive definite and thus non singular, the product $\left(\dot{\chi}_{k}(\theta)-\right.$ $\left.R^{-1} N^{T} \xi_{k}(\tau)\right)^{T} R\left(\dot{\chi}_{k}(\theta)-R^{-1} N^{T} \xi_{k}(\tau)\right)$ is positive for all $\tau \in$ $\left[0, T_{k}\right.$ [ and all $\theta \in[0 \tau[$. Integrating its development over $[0 \tau]$, the following inequality is obtained

$$
\begin{array}{r}
\int_{0}^{\tau} \dot{\chi}_{k}^{T}(\theta) R \dot{\chi}_{k}(\theta) d \theta-2 \xi_{k}^{T}(\tau) N \zeta_{k}(\tau) \\
+\tau \xi_{k}^{T}(\tau) N R^{-1} N^{T} \xi_{k}(\tau) \geq 0,
\end{array}
$$

Combining this inequality to equation (22), it yields:

$$
\begin{aligned}
\mathscr{W}\left(\tau, \chi_{k}\right) & \leq 2 \chi_{k}(\tau)^{T} P \dot{\chi}_{k}(\tau)+\left(T_{k}-2 \tau\right) \chi_{k}^{T}(0) X \chi_{k}(0) \\
& -\zeta_{k}^{T}(\tau)\left[S_{1} \zeta_{k}(\tau)+2 S_{2} \chi_{k}(0)\right]-2 \xi_{k}^{T}(\tau) N \zeta_{k}(\tau) \\
& +\left(T_{k}-\tau\right) \dot{\chi}_{k}^{T}(\tau)\left[R \dot{\chi}_{k}(\tau)+2 S_{1} \zeta_{k}(\tau)+2 S_{2} \chi_{k}(0)\right]
\end{aligned}
$$

In order to achieve the design of the controller the vector $\dot{\chi}_{k}(\tau)$ is introduced in the vector $\xi_{k}(\tau)$. Consequently, the exists a relation between the components of $\xi_{k}(\tau)$. This relation is given by:

$$
M_{0} \xi_{k}(\tau)=A \chi_{k}(\tau)-\dot{\chi}_{k}(\tau)+B K \chi_{k}(0)-B \psi\left(K \chi_{k}(0)\right)=0
$$

This allows to introduce the following terms to the inequality (23) for any square matrice $Y_{1}$ and $Y_{2} \in \mathbb{R}^{n \times n}$

$$
2\left[\chi_{k}^{T}(\tau) Y_{1}^{T}+\dot{\chi}_{k}^{T}(\tau) Y_{2}^{T}\right] M_{0} \xi_{k}(\tau)=0
$$

This method corresponds to the descriptor approach introduced in Fridman and Shaked (2002).

Suppose now that $\chi_{k}(0) \in \mathscr{S}$ defined in (12). In this case, from Lemma 2, the relation $-\psi^{T}\left(K \chi_{k}(0)\right) U\left[\psi\left(K \chi_{k}(0)\right)-\right.$ $\left.G \chi_{k}(0)\right] \geq 0$ holds. Define

$\mathscr{V}\left(\tau, \chi_{k}\right)=\mathscr{W}\left(\tau, \chi_{k}\right)-2 \psi^{T}\left(K \chi_{k}(0)\right) U\left[\psi\left(K \chi_{k}(0)\right)-G \chi_{k}(0)\right]$

Hence, if $\mathscr{V}\left(\tau, \chi_{k}\right)<0$, it follows that $\mathscr{W}\left(\tau, \chi_{k}\right) \leq \mathscr{V}\left(\tau, \chi_{k}\right)<$ 0 . The expression of $\mathscr{V}\left(\tau, \chi_{k}\right)$ is therefore given by

$$
\begin{aligned}
\mathscr{V}\left(\tau, \chi_{k}\right) & =\mathscr{W}\left(\tau, \chi_{k}\right)-2 \psi^{T}\left(K \chi_{k}(0)\right) U\left[\psi\left(K \chi_{k}(0)\right)-G \chi_{k}(0)\right] \\
\mathscr{V}\left(\tau, \chi_{k}\right) \leq & 2 \chi_{k}^{T}(\tau) P \dot{\chi}_{k}(\tau)+\left(T_{k}-2 \tau\right) \chi_{k}^{T}(0) X \chi_{k}(0) \\
& -\zeta_{k}^{T}(\tau)\left[S_{1} \zeta_{k}(\tau)+2 S_{2} \chi_{k}(0)\right] \\
& +\tau \xi_{k}^{T}(\tau) N R^{-1} N^{T} \xi_{k}(\tau)-2 \xi_{k}^{T}(\tau) N \zeta_{k}(\tau) \\
& +2 \xi_{k}^{T}(\tau) M_{0}^{T}\left(Y_{1} \chi_{k}(\tau)+Y_{2} \dot{\chi}_{k}(\tau)\right) \\
& +\left(T_{k}-\tau\right) \dot{\chi}_{k}^{T}(\tau)\left[R \dot{\chi}_{k}(\tau)+2 S_{1} \zeta_{k}(\tau)+2 S_{2} \chi_{k}(0)\right] \\
& -2 \psi\left({ }^{T} K \chi_{k}(0)\right) U\left[\psi\left(K \chi_{k}(0)\right)-G \chi_{k}(0)\right] .
\end{aligned}
$$

Noting that $\chi_{k}(\tau)=M_{1} \xi_{k}(\tau), \chi_{k}(0)=M_{2} \xi_{k}(\tau), \dot{\chi}_{k}(\tau)=$ $M_{3} \xi_{k}(\tau), \psi\left(K \chi_{k}(0)\right)=M_{4} \xi_{k}(\tau), \zeta_{k}=M_{12} \xi_{k}(\tau)$, we can rewrite $\mathscr{V}\left(\tau, \chi_{k}\right), \forall \tau \in\left[0 T_{k}[\right.$, as follows:

$$
\begin{aligned}
\mathscr{V}\left(\tau, \chi_{k}\right) \leq & \xi_{k}^{T}(\tau)\left[\Pi_{1}+\left(T_{k}-\tau\right) \Pi_{2}\right. \\
& \left.+\tau N R^{-1} N^{T}+\left(T_{k}-2 \tau\right) \Pi_{3}\right] \xi_{k}(\tau) .
\end{aligned}
$$

where

$$
\begin{aligned}
\Pi_{1} & =\operatorname{He}\left\{M_{1}^{T} P M_{3}-\left(M_{2}^{T} S_{2}^{T}-N\right) M_{12}\right\}-M_{12}^{T} S_{1} M_{12} \\
& +\operatorname{He}\left\{M_{0}^{T}\left(Y_{1} M_{1}+Y_{2} M_{3}\right)+M_{4}^{T} U G M_{2}\right\}-2 M_{4}^{T} U M_{4}, \\
\Pi_{2} & =M_{3}^{T} R M_{3}+\operatorname{He}\left\{M_{3}^{T}\left(S_{1} M_{12}+S_{2} M_{2}\right)\right\} \\
\Pi_{3} & =M_{2}^{T} X M_{2},
\end{aligned}
$$

In order to obtain an LMI condition that allows to compute a gain $K$, while guaranteeing that $\mathscr{V}\left(\tau, \chi_{k}\right)<0$, a method inspired from Fridman (2006) is applied in the sequel. 
Consider the contributions in $\mathscr{V}$ corresponding to terms of the form “ $\dot{\chi}_{k}^{T}(\tau)(.) \dot{\chi}_{k}(\tau)$ ”. This is given by

$$
M_{3}\left[\Pi_{1}+\left(T_{k}-\tau\right) \Pi_{2}+\tau N R^{-1} N^{T}+\left(T_{k}-2 \tau\right) \Pi_{3}\right] M_{3}^{T} .
$$

This leads to $-Y_{2}-Y_{2}^{T}+\tau R+\tau N_{3} R^{-1} N_{3}^{T}$, where $N_{3} \in \mathbb{R}^{n \times n}$ is a component of $N$. Since we are looking for a negative contribution of this term, a necessary conditions is that $Y_{2}$ is non singular. Thus, since $U$ is assumed to be a diagonal positive definite matrix, define the matrices $\tilde{Y}=Y_{2}^{-1}, \tilde{U}=U^{-1}$ and the matrix $\Xi=\operatorname{diag}\{\tilde{Y}, \tilde{Y}, \tilde{Y}, \tilde{U}\}$. This matrice $\Xi$ exists and is non singular. Introduce the new vector $\tilde{\xi}_{k}(\tau)=\Xi^{-1} \xi_{k}(\tau)$. Rewriting (24), using the new variable $\tilde{\xi}_{k}(\tau)$ leads to

$$
\begin{aligned}
\mathscr{V}\left(\tau, \chi_{k}\right) \leq & \tilde{\xi}_{k}^{T}(\tau)\left[\Xi^{T} \Pi_{1} \Xi+\left(T_{k}-\tau\right) \Xi^{T} \Pi_{2} \Xi\right. \\
& \left.+\tau \Xi^{T} N R^{-1} N^{T} \Xi+\left(T_{k}-2 \tau\right) \Xi^{T} \Pi_{3} \Xi\right] \tilde{\xi}_{k}(\tau) .
\end{aligned}
$$

The following consists in showing that the conditions of Theorem 2 leads to $\mathscr{V}\left(\tau, \chi_{k}\right)<0$. From the definition of the matrices $M_{i}$ for $i=1, \ldots, 4$, one has

$$
\begin{aligned}
& M_{1} \Xi=\tilde{Y} M_{1}, M_{2} \Xi=\tilde{Y} M_{2}, M_{3} \Xi=\tilde{Y} M_{3}, \\
& M_{4} \Xi=\tilde{U} M_{4}, M_{12} \Xi=\tilde{Y} M_{12} .
\end{aligned}
$$

Consequently, it yields

$$
\begin{gathered}
\Xi^{T} \Pi_{1} \Xi=\operatorname{He}\left\{M_{1}^{T} \tilde{Y}^{T} P \tilde{Y} M_{3}-\left(M_{2}^{T} \tilde{Y}^{T} S_{2}^{T} \tilde{Y}-\Xi^{T} N \tilde{Y}\right) M_{12}\right\} \\
-M_{12}^{T} \tilde{Y}^{T} S_{1} \tilde{Y} M_{12}+\operatorname{He}\left\{\left(M_{1}^{T} \tilde{Y}^{T} Y_{1}^{T}+M_{3}^{T}\right) M_{0} \Xi\right\} \\
-2 M_{4}^{T} \tilde{U} M_{4}+\operatorname{He}\left\{M_{4}^{T} G \tilde{Y} M_{2}\right\},
\end{gathered}
$$$$
\Xi^{T} \Pi_{2} \Xi=M_{3}^{T} \tilde{Y}^{T} R \tilde{Y} M_{3}+2 \operatorname{He}\left\{M_{3}^{T}\left(\tilde{Y}^{T} S_{1} \tilde{Y} M_{12}+\tilde{Y}^{T} S_{2} \tilde{Y} M_{2}\right)\right\},
$$$$
\Xi^{T} \Pi_{3} \Xi=M_{2}^{T} \tilde{Y}^{T} X \tilde{Y} M_{2},
$$

Define the new LMI variables $\tilde{P}=\tilde{Y}^{T} P \tilde{Y}, \tilde{S}_{1}=\tilde{Y}^{T} S_{1} \tilde{Y}, \tilde{S}_{2}=$ $\tilde{Y}^{T} S_{2} \tilde{Y}, \tilde{R}=\tilde{Y}^{T} R \tilde{Y}, \tilde{X}=\tilde{Y} T \tilde{Y}$ and $\tilde{N}=\Xi^{T} N \tilde{Y}$. With these notations, the non linear term $\Xi^{T} N R^{-1} N^{T} \Xi$ can be rewritten as follows:

$$
\begin{aligned}
\Xi^{T} N R^{-1} N^{T} \Xi & =\Xi^{T} N \tilde{Y}\left(\tilde{Y}^{-1} R^{-1} \tilde{Y}^{-T}\right) \tilde{Y}^{T} N^{T} \Xi \\
& =\tilde{N} \tilde{R}^{-1} \tilde{N} .
\end{aligned}
$$

From the definition of $\tilde{Y}$, we have $\tilde{Y}^{T} Y_{2}=I$. Concerning the term $\tilde{Y}^{T} Y_{1}$, it is necessary to introduce the constraint $Y_{1}=\varepsilon Y_{2}$, for some positive scalar $\varepsilon$. Define $\tilde{K}=K \tilde{Y}$ and $\tilde{G}=G \tilde{Y}$, the following inequality is thus obtained

$$
\begin{aligned}
\mathscr{V}\left(\tau, \chi_{k}\right) \leq & \tilde{\xi}_{k}^{T}(\tau)\left[\tilde{\Pi}_{1}+\left(T_{k}-\tau\right) \tilde{\Pi}_{2}\right. \\
& \left.+\tau \tilde{N} \tilde{R}^{-1} \tilde{N}^{T}+\left(T_{k}-2 \tau\right) \tilde{\Pi}_{3}\right] \tilde{\xi}_{k}(\tau) .
\end{aligned}
$$

where $\tilde{\Pi}_{1}, \tilde{\Pi}_{2}$ and $\tilde{\Pi}_{3}$ are defined in (20). To prove that $\mathscr{V}$ is negative definite for all $\tau$, note that the right hand side of equation (25) depends linearly on $\tau$ which belongs to the interval $\left[0, T_{k}\right]$. A convexity property ensure that this is equivalent to only ensure that this inequality holds for $\tau=0$ and $\tau=T_{k}$ (see Naghshtabrizi et al. (2008) for more detail). It leads to the inequalities

$$
\begin{aligned}
& \tilde{\Pi}_{1}+T_{k}\left(\tilde{\Pi}_{2}+\tilde{\Pi}_{3}\right)<0, \\
& \tilde{\Pi}_{1}-T_{k} \tilde{\Pi}_{3}+T_{k} \tilde{N} \tilde{R}^{-1} \tilde{N}^{T}<0 .
\end{aligned}
$$

Applying the same convexity on $T_{k}$ lying in the interval $\left[\bar{T}_{1}, \bar{T}_{2}\right]$ and then applying the Schur complement to the non linear term, the conditions $\Psi^{1}\left(\bar{T}_{i}\right)<0$ and $\Psi^{2}\left(\bar{T}_{i}\right)<0$ are obtained.

Hence, we can conclude that if $\Psi^{1}\left(\bar{T}_{i}\right)<0, \Psi^{2}\left(\bar{T}_{i}\right)<0$ and provided that $\chi_{k}(0) \in \mathscr{S}$, it follows that $W\left(\tau, \chi_{k}\right)<0$ and the conditions of Theorem 1 are verified. We show now that, if $x_{0}=\chi_{0}(0)$ is in $\mathscr{E}=\left\{x \in \mathbb{R}^{n}, \quad x^{T} P x \leq 1\right\}$ and if (19) is verified, it follows that $W\left(\tau, \chi_{k}\right)<0, \forall k \geq \overline{0}$.
It is easy to see that (19) implies that $\mathscr{E} \subset \mathscr{S}$ as defined in (12). Suppose that $\chi_{0}(0) \in \mathscr{E}$. Hence it follows, from Lemma 2 that $\psi^{T}\left(K \chi_{0}(0)\right) U\left[\psi\left(K \chi_{0}(0)\right)-G \chi_{0}(0)\right] \leq 0$ and therefore, if $\Psi^{1}\left(\bar{T}_{i}\right)<0, \Psi^{2}\left(\bar{T}_{i}\right)<0, \mathscr{W}\left(\tau, \chi_{0}\right)<0$. This fact implies that

$$
V\left(\chi_{1}(0)\right)=V\left(\chi_{0}\left(T_{1}\right)\right)<V\left(\chi_{0}(0)\right)
$$

i.e. $\chi_{1}(0)$ is in $\mathscr{E}$. Repeating the reasoning for $k=1,2 \ldots$, we conclude that $\chi_{l}(0) \in \mathscr{E}$ and $\psi^{T}\left(K \chi_{k}(0)\right) U\left[\psi\left(K \chi_{k}(0)\right)-\right.$ $\left.G \chi_{k}(0)\right] \leq 0, \forall k=[0, \infty)$. Hence, by vertu of Theorem 1 the satisfaction of conditions (17)-(19) ensures the asymptotic convergence of the trajectories to the origin, provided that $x_{0} \in \mathscr{S}$.

A remark regarding Theorem 2 concerns the traduction of the criteria in terms of the matrices defining the discrete-time system (6). If the stabilization criteria is satisfied, then, by virtue of Theorem 1, it yields

$\forall T_{k} \in\left[\bar{T}_{1}, \bar{T}_{2}\right]$ and $\forall x_{0} \in \mathscr{E}$,

$$
\Delta V(k)=x_{k}^{T}\left(\tilde{A}^{T}\left(T_{k}\right) P \tilde{A}\left(T_{k}\right)-P\right) x_{k}+2 x_{k} P \tilde{B}\left(T_{k}\right) \operatorname{sat}\left(K x_{k}\right)<0,
$$

with the corresponding $K$ and the matrices $\tilde{A}\left(T_{k}\right)$ and $\tilde{B}\left(T_{k}\right)$ given in (6).

\section{OPTIMIZATION ALGORITHMS}

From the theoretical result given by Theorem 2, this section provides the formulation of some optimization algorithms to address the stabilization problems (P1) and (P2) defined in Section 2.

\subsection{Maximization of the estimate of the region of attraction}

For a given $u_{0}$ and an asynchronous sampling satisfying (1) with given $\bar{T}_{1}$ and $\bar{T}_{2}$, the objective is to determine $K$ that leads to largest set of of initial conditions for which the conditions of Theorem 2 are satisfied. In other words, we should find $K$ such that $\mathscr{E}$ is maximized considering some size criterion. For instance, we can maximize the minimal axis of $\mathscr{E}$, which corresponds to minimize the maximal eigenvalue of $P$. This can be accomplished from the following optimization problem

$$
\begin{gathered}
\max \delta \\
\text { subject to } \\
(17),(18),(19) \\
{\left[\begin{array}{cc}
-\delta I & I \\
I & -\tilde{Y}-\tilde{Y}^{T}+\tilde{P}
\end{array}\right]<0 .}
\end{gathered}
$$

Note that, from Lemma 1 , the last inequality above ensures that $P<\delta I$, which ensures that $\lambda_{\max }(P)<\delta$.

\subsection{Maximization of the sampling period}

Define a region of admissible initial states

$$
\mathscr{E}_{0}=\left\{x \in \mathbb{R}^{n}, \quad x^{T} P_{0} x \leq 1\right\} .
$$

Hence, given $\bar{T}_{1}$ the idea is to find the maximal $\bar{T}_{2}$ for which it is possible to compute $K$ such that the asymptotic stability is ensured for all initial conditions belonging to $\mathscr{E}_{0}$. An upperbound on the maximal $\bar{T}_{2}$ can therefore be obtained from the following optimization problem 


$$
\begin{gathered}
\max \bar{T}_{2} \\
\text { subject to } \\
(17),(18),(19) \\
{\left[\begin{array}{cc}
-P_{0} & I \\
I & -\tilde{Y}-\tilde{Y}^{T}+\tilde{P}
\end{array}\right]<0 .}
\end{gathered}
$$

Note that, from Lemma 1, the last inequality above ensures that $P<P_{0}$, which ensures that $\mathscr{E}_{0} \subset \mathscr{E}$.

Remark 2. Note that, for a fixed $\varepsilon$, the constraints in (26) and (27) are LMIs. Then the optimal solution can be easily approached by solving LMI-based problems on a grid in $\varepsilon$.

\section{EXAMPLES}

We consider the system (2) with the following matrices (taken from Cao et al. (2002)):

$$
A=\left[\begin{array}{ll}
1.1 & -0.6 \\
0.5 & -1.0
\end{array}\right], \quad B=\left[\begin{array}{l}
1 \\
1
\end{array}\right],
$$

and where $\bar{u}=5$. From Fridman et al. (2004), the gain $K=$ $\left[\begin{array}{ll}-1.696 & 0.523\end{array}\right]$ ensures the local stability of the closed loop for all samplings with the maximum sampling interval $T \leq 0.75$ and the set of admissible initial conditions is defined by

$$
x_{0}^{T}\left[\begin{array}{rr}
0.9132 & -0.2816 \\
-0.2816 & 0.0868
\end{array}\right] x_{0} \leq 1 .
$$

The largest circle included in this ellipsoid is of radius 0.3551 .

Based on Theorem 2, the gain $K=\left[\begin{array}{ll}2.037 & -0.628\end{array}\right]$ ensures the stability of the system under any asynchronous sampling period satisfying (1) with $\bar{T}_{1}=0$ and $\bar{T}_{2}=0.75$ for all initial conditions is defined by

$$
x_{0}^{T}\left[\begin{array}{rr}
0.0807 & -0.0249 \\
-0.0249 & 0.0077
\end{array}\right] x_{0} \leq 1,
$$

and the larger circle included in this ellipsoid is given by ||$x_{0} \| \leq 3.391$ (corresponding to $\delta=90.1 \times 10^{-3}$ ) which is approximatively ten times larger than the one from Fridman et al. (2004). Moreover, it is possible to design a stabilizing state control feedback for all asynchronous samplings satisfying with $\bar{T}_{1}=0$ and $\bar{T}_{2}=1.38$. This proves the efficiency of the method.

\section{CONCLUSION}

This article proposed a novel constructive stabilization condition for sampled data control systems taking into account sampling period variation and actuators constraints. The basic idea behind the method consists in considering a continuous functional whose values at the sampling instants coincides with a quadratic Lyapunov function for the discrete-time system. The actuator saturation is tackled using a generalized sector condition. The derived conditions are in LMI form, which allows to compute a stabilizing state feedback and an associated set of admissible initial conditions by solving convex optimization problems. The method is therefore suitable to cope with sampling jitter and package losses in networked control systems.

\section{REFERENCES}

Cao, Y., Lin, Z., and Hu, T. (2002). Stability analysis of linear time-delay systems subject to input saturation. IEEE Trans. on Circ. and Syst. I, 49, 233-240.

Fridman, E. (2006). Descriptor discretized Lyapunov functional method: Analysis and design. IEEE Trans. on Automatic Control, 51(5), 890-897.
Fridman, E. (2010). A refined input delay approach to sampleddata control. Automatica, 46(2), 421-427.

Fridman, E., Seuret, A., and Richard, J.P. (2004). Robust sampled-data stabilization of linear systems: an input delay approach. Automatica, 40(8), 1441-1446.

Fridman, E. and Shaked, U. (2002). A descriptor system approach to $H^{\infty}$ control of linear time-delay systems. IEEE Trans. on Automatic Control, 47(2), 253-270.

Gomes da Silva Jr., J.M., Seuret, A., Fridman, E., and Richard, J.P. (2010). Stabilisation of neutral systems with saturating control inputs. Int. J. Syst. Sci., iFirst, DOI: 10.1080/00207720903353575.

Gomes da Silva Jr., J.M. and Tarbouriech, S. (2005). Antiwindup design with guaranteed regions of stability: an LMIbased approach. IEEE Trans. Autom. Contr., 50(1), 106-111.

Hespanha, J., Naghshtabrizi, P., and Xu, Y. (2007). A survey of recent results in networked control systems. Proceedings of the IEEE, 95(1), 138-162.

Khalil, H.K. (1996). Nonlinear Systems. Prentice Hall.

Lin, Z. and Saberi, A. (1993). Semi-global exponential stabilization of linear systems subject to input saturation via linear feedback. Syst. \& Contr. Lett., 21, 225-239.

Naghshtabrizi, P., Hespanha, J., and Teel, A. (2008). Exponential stability of impulsive systems with application to uncertain sampled-data systems. Systems and Control Letters, 57(5), 378-385.

Niculescu, S.I., Dion, J.M., and Dugard, L. (1996). Robust stabilization for uncertain time-delay systems containing saturating actuators. IEEE Trans. Autom. Contr., 41(5), 742747.

Oucheriah, S. (1996). Global stabilization of a class of linear continuous time delay systems with saturating controls. IEEE Trans. on Circ. and Syst. I, 43(12), 1012-1015.

Peet, M., Papachristodoulou, A., and Lall, S. (2009). Positive forms and stability of linear time-delay systems. SIAM Journal on Control and Optimization, 47(6), 3227-3258.

Seuret, A. (2011). A novel stability analysis of linear systems under asynchronous samplings. Accepted for publication to Automatica.

Tarbouriech, S. and Gomes da Silva Jr., J.M. (2000). Synthesis of controllers for continuous-time delay systems with saturating controls via LMIs. IEEE Trans. Autom. Contr., 45(1), 105-111.

Tarbouriech, S., Gomes da Silva Jr., J., and Garcia, G. (2003). Delay-dependent anti-windup loops for enlarging the stability region of time-delay systems with saturating inputs. Trans. ASME - J. of Dyn. Syst., Meas.and Contr., 125(1), 265-267.

Zampieri, S. (2008). A survey of recent results in Networked Control Systems. In Proc. of the $17^{\text {th }}$ IFAC World Congress. Seoul, Korea.

Zhang, L., Boukas, E., and Haidar, A. (2008). Delay-rangedependent control synthesis for time-delay systems with actuator saturation. Automatica, 44, 26912695. 